\title{
COMPARATIVE ANALYSIS OF HYPOCHLORITE METHOD ON TRANSGENIC STRAIN BY250
}

\author{
Neha Singh and Priyanka Gautam \\ Bioinformatics Lab, Dept. of Zoology Dayalbagh Educational Institute, \\ Dayalbagh, Agra-282 005, India.nehasingh.kain@gmail.com
}

\begin{abstract}
Caenorhabditis elegans is an ideal model organism that has been applied to a wide range of studies into toxicology, neurobiology, molecular, and developmental biology. C.elegans has gain popularity as a model system worldwide because of its availability of genome database and similarity of the genome to human have enlarged its applications in the different areas of research and to study the molecular mechanism of various human diseases such as neurodegenerative, metabolic, and so on. The researchers were synchronized C.elegans to larval stage L1 before they experimented. These studies were propagated and describe as a powerful tool to obtain a large population of worms through a synchronization process at a specific stage, which can be used for a variety of experiments.
\end{abstract}

KEYWORDS: Caenorhabditis elegans, Hypochlorite Solution, Transgenic Worm BY250, Synchronization

\section{INTRODUCTION}

C.elegans is a free-living, naturally lives in a temperate soil environment with small body size, short life span, easy to culture in the laboratory, ease of observation, inexpensive in cultivation in the laboratory settings and feeds on E.coli OP50 bacterial food source. C.elegans is the first multicellular model organism that has a completely sequenced genome ${ }^{1}$. Scientist Sydney Brenner was first introduced as a model organism into research in $1974{ }^{5}$. After the introduction of this model organism by Brenner, this is used in many different areas such as neurobiology, developmental biology, toxicology, and molecular biology also. The tough but transparent cuticle of C.elegans allows us to visualize internal structures without dissection and specific gene expressions ${ }^{4}$. As we describe that C.elegans have ortholog genes linked to humans, many genes linked to various neurodegenerative diseases exist in this nematode including Parkinson's disease-related genes like pdr-1, pink-1, djr1.1,1.2, lrk- ${ }^{8}$. C.elegans have different strains like wild type, mutant, and transgenic, the transgenic approaches have also been used to model neurodegenerative diseases such as Parkinson's, Alzheimer's, muscular dystrophy, and Amyotrophic lateral sclerosis ${ }^{2}$. The transgenic nematodes were obtained by micro-injection technique ${ }^{6}$. These worms were stained with GFP (Green Fluorescent Protein) with tagged transporter promoter dat-1::GFP. In this protocol, we illustrated and explain that how we obtained the age synchronized worms population from a large number of worms by using the alkaline hypochlorite method before performing the chemical treatment. 


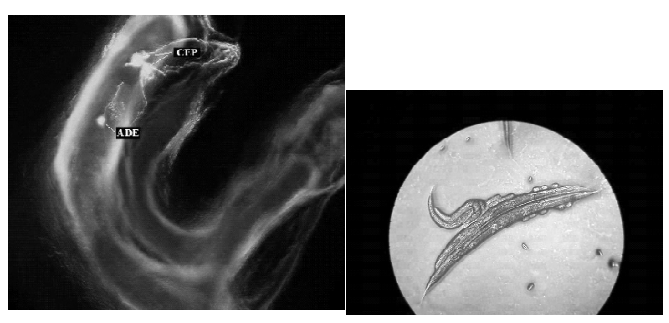

(a)

(b)

Figure: 1 (a) Adult N2 worm (b) Adult Transgenic BY250 worm.

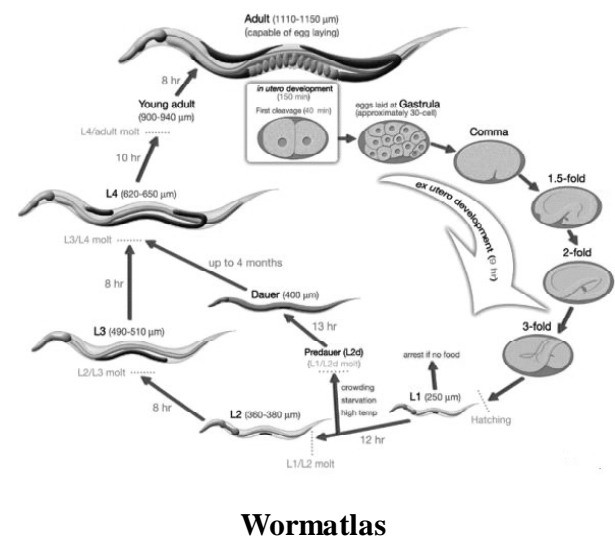

Figure: 2 - Life cycle of C.elegans at $22^{\circ} \mathrm{C}$.

\section{MATERIALS AND METHODS}

Reagents used in nematode culture and synchronization

The transgenic strain BY250 was a kind gift of Dr. Randy Blakely was acquired from, The Blakely Lab, Charles E. Schmidt College of Medicine, Brain Institute, Florida Atlantic University. E.coli OP50 can be obtained from the Caenorhabditis Genetics Center (CGC) Minnesota University, USA.

1. Nematode Growth Medium: $\mathrm{NaCl}$, Bacto-peptone, Agar, Cholesterol, $1 \mathrm{M}$ $\mathrm{CaCl}^{2}, 1 \mathrm{M} \mathrm{MgSO} 4$ in $\mathrm{dH}_{2} \mathrm{O}$
2. E.coli OP50: $2 \mathrm{gm}$ of Bacto - agar in $20 \mathrm{ml}$ of $\mathrm{dH}_{2} \mathrm{O}$

3. Bleaching solution: $3 \mathrm{gm}$ of $5 \mathrm{M} \mathrm{NaOH}$

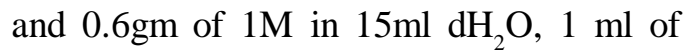
$4 \% \mathrm{NaOCl}$.

5. M9 Buffer: $\mathrm{KH}_{2} \mathrm{PO}_{4}, \mathrm{Na}_{2} \mathrm{HPO}_{4}, \mathrm{NaCl}, 1$ $\mathrm{M} \mathrm{MgSO}_{4}$ in $\mathrm{dH}_{2} \mathrm{O}$. Sterilized by autoclaving

\section{Culturing of unsynchronized BY250} worms for synchronization

Transgenic strains were used to address some specific experiments because the neurons are stained with dyes through which we can detect or discriminate the diseasespecific neurons. The BY250 worms are handle as N2 wild-type strain. The large number of populations of BY250 can be obtained by culturing them on solid NGM (Nematode Growth Media) and fed with E.coli OP5O bacteria which is added to the plates (Riva et.al., 2012, Stiernagle, 2006).

PROTOCOL: 1 PREPARATION OF NGM MEDIA AND PETRI PLATES

- Weighing $\mathrm{NaCl}$, Agar, and Peptone in a conical flask and add $\mathrm{dH}_{2} \mathrm{O}$. Keep this flask into the autoclave for $50 \mathrm{~min}$.

- After autoclaving cool the flask to $55^{\circ} \mathrm{C}$ and then add $1 \mathrm{M} \mathrm{CaCl}_{2}, 5 \mathrm{mg} / \mathrm{ml}$ cholesterol in ethanol, $1 \mathrm{M} \mathrm{MgSO}_{4}$ and $1 \mathrm{M} \mathrm{KPO}_{4}$ buffer (Do not autoclave all these ingredients).

- Using sterile procedures dispense the NGM solution into Petri plates.

- Once the plates was dry we were leave plates at $37 \ddot{\mathrm{A} C}$ for $24 \mathrm{hr}$ in incubator 
before use for detection of contaminants.

- After 24h plates was streak with E.coli OP50 in the laminar air flow. We were pick a single colony and grow overnight at room temperature in incubator

- Allow the E.coli OP50 lawn to grow overnight and after this add the desired amount of worms to the plates and leave it for 3 days at $22^{\circ} \mathrm{C}$ in BOD incubator to grow an adult stage.

\section{Harvesting BY250 worm with Alkaline Hypochlorite Solution (Bleaching)}

In our work, we have performed a synchronization method on freshly grown gravid nematode population with $1 \mathrm{M}$ and
$5 \mathrm{M}$ of bleaching solution with both the strains of BY250 and N2 wild type also. The bleaching technique is used for synchronizing C.elegans cultures at the first larval stage (L1). If we fed the worms with E.coli OP50 the L1 worms were grown to an adult stage but when we were not fed the worms the L1 stage was remain in this stage which allows hatching but prevents further development. So the principle of this method lies in the fact that worms are sensitive to bleach, while the eggshell protects embryos from it. After treatment with alkaline hypochlorite solution, the eggshell was ruptured and embryos came outside from the worm's body.

Table-1: Ratio used for bleaching Solution for hypochlorite method

\begin{tabular}{|l|c|c|}
\hline & Bleaching Solution (1M) & Bleaching Solution (5M) \\
\hline Water $(\mathbf{m l})$ & 0.5 & 3.5 \\
\hline Sodium hydroxide $(\mathbf{m l})$ & 2.5 & 0.5 \\
\hline Sodium hypochlorite $\sim \mathbf{4 \%}(\mathbf{m l})$ & 1 & 1 \\
\hline Total $(\mathbf{m l})$ & 4 & 5 \\
\hline
\end{tabular}

PROTOCOL: 2 SYNCHRONIZATION METHOD

- Allow worms to grow until an adult stage.

- Gravid adult worms harvested from the cultured plate by rinsing the plate with M9 buffer and collect in $15 \mathrm{ml}$ centrifuge tubes.

- The worms were settle down in the centrifuge tube then the pelleted worms were collected by centrifuging for 2 minutes at $\sim 1500 \mathrm{rpm}$ on a standard table centrifuge at room-temperature and discard supernatant.

- The worm pellet was washed 3-4 times with M9 buffer until the buffer appears clear of bacteria.

- After clear all the debris from the pellet add the desired bleaching solution (Table 1).

- Agitate the tube for some minutes and the destruction and dissociation of the adult egg shell can be monitored under the dissecting microscope with 10x lens. 
- The reaction was stopped when we were found that the eggs came outside from the worm's body by the bursting of egg shell which typically takes between 3 and 5 minutes.

- The reaction was stopped by adding M9 buffer to fill the centrifuge tube.

- Quickly centrifuge for 1 minute at $400 x g$ $(\sim 1500 \mathrm{rpm})$ and aspirate most of the M9 buffer without disturbing the worm's pellet.

- Wash the worms pellet 1-2 times with M9 buffer and centrifuge each and every time.

- Add $0.5 \mathrm{ml}$ or $1 \mathrm{ml}$ of M9 (According to the number of synchronized worms you needed) buffer to the pellet and place this solution to the seeded NGM plates.

- Then incubate the plate at the $22^{\circ} \mathrm{C}$ in BOD incubator and leave it for 3 days to grow an adult stage.

\section{PRECAUTIONS}

- When you will add the bleaching solution into the worm pellet after washing with M9 buffer the time should not be exceeded because the cuticle of C.elegans is more sensitive to the hypochlorite solution. So it may be chances that the eggs will also be killed. So after examining the bursting of eggshell quickly add M9 buffer to stop the reaction of hypochlorite solution. So do not bleach for much longer time unless you will kill the eggs also.

- Stop the reaction when $60-80 \%$ of worms are broken in the middle.
- If there is no food on the plate the larvae should be halted at the L1 larval stage.

- Wash the pellet with M9 buffer until the buffer appears clear of bacteria.

- The Bleaching solution was made fresh and must be performed just before its use.

\section{RESULTS AND DISCUSSION}

The synchronous population of worms may be needed for some experiments to eliminate variation in results due to age differences. Eggs are resistant to bleach and after several washes, they are collected and transferred to new NGM plates that generating the new synchronous population. The ease of maintaining this nematode added to the possibility of obtaining synchronized populations of worms at a specific stage through a relatively easy protocol an additional feature that makes C.elegans an attractive model to work with is the possibility. We have performed two hypochlorite methods to obtain a synchronous population of worms one is with $1 \mathrm{M}$ and the other with $5 \mathrm{M}$ of bleaching solution.

\section{Comparison between $1 \mathrm{M}$ and $\mathbf{5 M}$}

We have done an age synchronization procedure with a $5 \mathrm{M}$ bleaching solution examine and monitored after day third under dissecting microscope. The eggs were hatched properly and grow into an adult stage properly. The movement of these worms was fast and this method was produced a large amount of age synchronous 
worm population. In the same laboratory conditions and temperature, we have also performed an alkaline hypochlorite method with a $1 \mathrm{~m}$ bleaching solution on a transgenic BY250 worm's population. The eggs were hatched and grow into a larval stage and the growth remained in the fourteen stages on day third. We have observed that the metamorphosis process was delayed in the worms treated with $1 \mathrm{M}$ alkaline hypochlorite solution that mean the process of conversion into an adult to the larval stage was delayed. This method of $1 \mathrm{~m}$ bleaching solution was also produced a good quantity of worm's population and showed normal movement. We analyzed our results and found that the worms treated with $1 \mathrm{M}$ bleaching solution were delayed in their development as compared to the $5 \mathrm{M}$ bleaching solution method. So from our results, we were found that the $5 \mathrm{M}$ bleaching solution method was best suited to obtain fourteen stages and adult after day third.

\section{Representative results}

Comparison of two different bleaching solutions at $22^{\circ} \mathrm{C}$ incubation times with exposure period of bleaching treatment is five minutes. BY250 transgenic worms were washed thrice with M9 were split or collected into two different $15 \mathrm{ml}$ centrifuge tubes containing two bleaching solutions. Tubes were shaken vigorously and $1 \mathrm{ml}$ transferred to a new tube and add M9 to stop the reaction after the specified time. After bleaching treatment worms were incubated with $1 \mathrm{ml}$ of $\mathrm{M} 9$ at $22^{\circ} \mathrm{C}$.

\section{Bleaching solution: 1 [1M]}

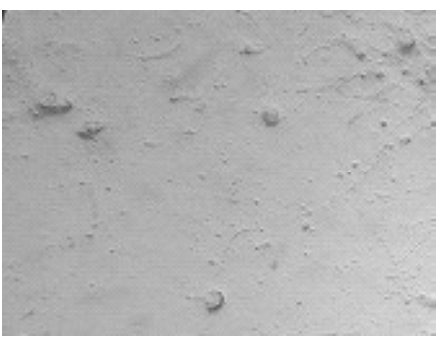

(A)

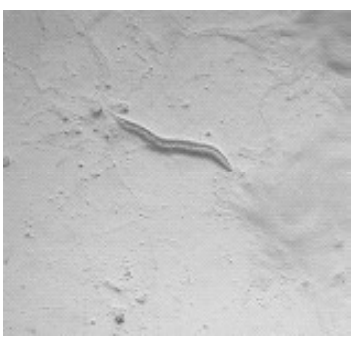

(B)

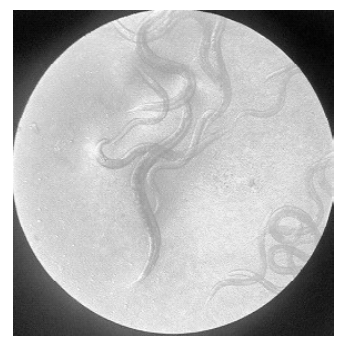

(C)

Figure 1: (A) Eggs of bleached transgenic nematode, picture was taken just after bleaching (B) Transgenic larval stage after the incubation period of $24 \mathrm{hr}$. at $22^{\circ} \mathrm{C}$ (C) Adult worms acquired after $92 \mathrm{hr}$. of incubation period. 


\section{Bleaching solution: 2 [5M]}

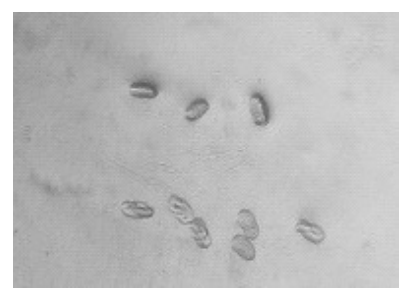

(A)

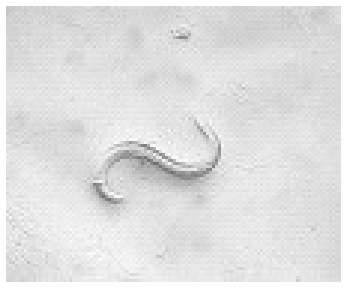

(B)

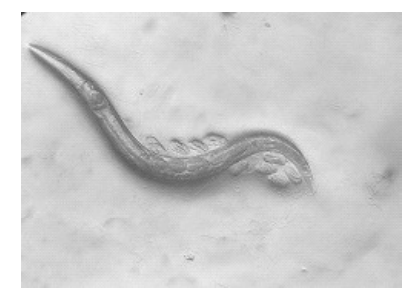

(C)

Figure 2: (A) Eggs of hypochlorite solution treatment worm just after bleaching process (B) Transgenic worm larval stage at $24 \mathrm{hr}$. in the same laboratory conditions (C) The adult worms obtained after $24 \mathrm{hr}$. of incubation period.

\section{AUTHOR'S CONTRIBUTION}

NS is the first author who carried out the experiments, statistically analyzed and interpreted the data and wrote the manuscript. PG was oversight responsibility for the research activity planning and acted as the corresponding author.

\section{CONFLICT OF INTEREST}

The authors state that they have no conflict of interest.

\section{REFERENCES}

- Bianch, L. \& M. Driscoll, 2006. Culture of embryonic C.elegans cells for electrophysiological and pharmacological analyses. Wormbook. 1-15.

- $\quad$ Boyd, W., M. Smith \& J.H. Freedman, 2012. Caenorhabditis elegans as a model in developmental toxicology. Meth Mol Biol., 889:15-24

- Fontrodona, L., A. Villanueva \& J. Cerón, 2012.
Basic Caenorhabditis elegans methods: synchronization and observation. Journal of Visualized Experiments, 64: 1-9.

- Hunt, Piper Reid, 2016. The C.elegans model in toxicity testing. Appl. Toxicol., 1-10. DOI: 10.1002/jat. 33:57.

- $\quad$ Leung, M.C.K., P.L. Williams, A. Benedetto, C. Au, K.J. Helmcke, M. Aschner \& J.N. Meyer, 2008. Caenorhabditis elegans: An emerging model in biomedical and environmental toxicology. Journal of Toxicological Sciences, 106: 5-28.

- Mullen, G.P., E.A. Mathews, M.H. Vu, J.W. Hunter, D.L. Frisby, A. Duke, K. Grundahl, J.D. Osborne, J.A. Crowell \& J.B. Rand, 2007. Choline Transport and de novo Choline Synthesis Support Acetylcholine. Genetics Society of America, 177: 195-204.

- $\quad$ Stiernagle, T., 2006. Maintenance of C.elegans. WormBook. doi/10.1895/Wormbook, 1.101. 1.

- Wolozin, B., C. Gabel, A. Ferree \& M. Guillily, 2011. Ebata, Atsushi Watching worms whither: Modeling neurodegeneration in C.elegans. Progress in Molecular Biology and Translational Science, 100: 499-514. 
